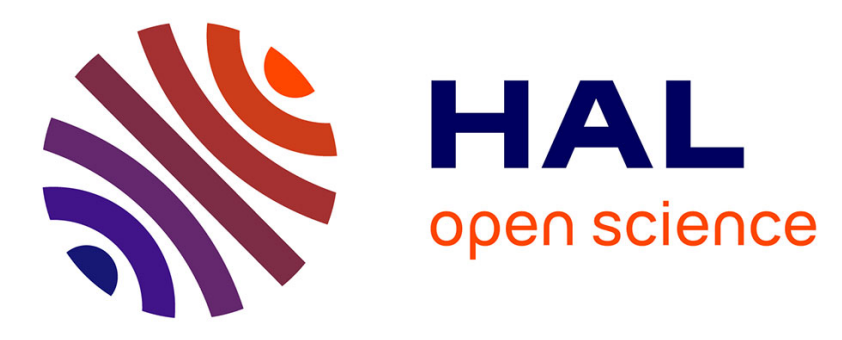

\title{
Instrumentation pour le suivi en ligne des traitements par hadronthérapie
}

V. Reithinger, L. Balleyguier, J. Baudot, M. Dahoumane, D. Dauvergne, S. Deng, N. Freud, J. Krimmer, J.-M. Letang, H. Mathez, et al.

\section{- To cite this version:}

V. Reithinger, L. Balleyguier, J. Baudot, M. Dahoumane, D. Dauvergne, et al.. Instrumentation pour le suivi en ligne des traitements par hadronthérapie. 6ème Colloque Interdisciplinaire en Instrumentation, C2I 2013, Jan 2013, Lyon, France. in press. hal-00838591

\section{HAL Id: hal-00838591 \\ https://hal.science/hal-00838591}

Submitted on 26 Jun 2013

HAL is a multi-disciplinary open access archive for the deposit and dissemination of scientific research documents, whether they are published or not. The documents may come from teaching and research institutions in France or abroad, or from public or private research centers.
L'archive ouverte pluridisciplinaire HAL, est destinée au dépôt et à la diffusion de documents scientifiques de niveau recherche, publiés ou non, émanant des établissements d'enseignement et de recherche français ou étrangers, des laboratoires publics ou privés. 


\title{
Instrumentation pour le suivi en ligne des traitements par hadronthérapie
}

V. Reithinger ${ }^{1}$, L. Balleyguier ${ }^{1}$, J. Baudot ${ }^{2}$, M. Dahoumane ${ }^{1}$, D. Dauvergne ${ }^{1}$, S. Deng ${ }^{1}$, N. Freud ${ }^{3}$, J. Krimmer ${ }^{1}$, J.-M. Letang ${ }^{3}$, H. Mathez ${ }^{1}$, G. Montarou 4 , C. Ray ${ }^{1}$, M.-H. Richard ${ }^{1}$, M. De Rydt ${ }^{5}$, E. Testa ${ }^{1}$, M. Winter ${ }^{2}$, Y. Zoccaratto ${ }^{1}$

1 Université de Lyon, UCBL, Institut de Physique Nucléaire de Lyon, Villeurbanne, France 2Université de Strasbourg, Institut Pluridisciplinaire Hubert Curien, Strasbourg, France 3Université de Lyon, CREATIS, Inserm, INSA-Lyon, Centre Léon Bérard, Lyon, France ${ }^{4}$ Univ. de Clermont-Ferrand, Lab. de Physique Corpusculaire, Clermont-Ferrand, France ${ }^{5}$ Physics Depart., InstituutvoorKern- en Stralingsfysica, KU Leuven, Leuven, Belgium

valerian.reithinger@ipnl.in2p3.fr

\author{
Abstract \\ Instrumentation for on-line monitoring of hadrontherapy treatments
}

Localization of the dose deposited in the patient is a key point for the hadrontherapy cancer treatment. Different modalities of on-line control are explored, motivating acquisition systems and detectors developments, such as a Compton gamma camera, a proton trajectometer and a beam hodoscope.

\section{Contexte}

\subsection{L'hadronthérapie}

Trois modalités de traitement du cancer sont couramment utilisées: la chirurgie, la chimiothérapie et la radiothérapie. Cette dernière consiste à irradier la zone tumorale avec des rayonnements ionisants. Leur interaction dans l'organisme mène à un dépôt d'énergie conduisant -via une cascade d'interactions physiques et biologiques- à la mort des cellules.

Alors que la radiothérapie externe exploite principalement des faisceaux de photons (environ $99 \%$ des cas), l'hadronthérapie [1] exploite des particules chargées telles que des protons ou des ions légers (carbone par exemple), dont les modes d'interaction physique sont différents.

Parmi les avantages de cette technique, on peut citer en premier lieu une meilleure conformation de l'irradiation à la zone tumorale, due à l'effet balistique des ions : le dépôt d'énergie, contrairement à celui des photons, est continu le long du parcours et présente un maximum en fin de parcours, lequel est bien déterminé par l'énergie de l'ion incident (pic de Bragg, voir la courbe du dépôt d'énergie présentée Figure 1).

Ce dépôt plus localisé dans l'espace permet de mieux épargner les tissus sains environnant la tumeur. De plus, comparativement aux photons et aux protons, I'hadronthérapie carbone se démarque par une efficacité biologique relative supérieure en fin de parcours. Le traitement des tumeurs radiorésistantes est alors plus efficace. 


\subsection{Contrôle en ligne}

\subsubsection{Nécessité}

La précision intrinsèque de l'hadronthérapie rend cette méthode d'autant plus sensible aux incertitudes qui peuvent conduire à une différence entre la planification de l'irradiation et la zone effectivement irradiée. II est donc nécessaire de contrôler en ligne, c'est-à-dire pendant le traitement -et si possible en temps réel- le bon déroulement de celui-ci.

\subsubsection{Principe}

Lors du parcours des ions dans l'organisme, deux types d'interactions peuvent se produire :

- lonisation, à l'origine du dépôt d'énergie et des effets biologiques ;

- Réactions nucléaires, menant à une activation nucléaire ou une émission de particules promptes telles que des rayons gammas, des neutrons, ou des protons.

La figure 1 représente, pour un faisceau d'ions carbone dans l'eau, les distributions longitudinales du dépôt de dose (noir), ainsi que les lieux d'interactions nucléaires (appelés vertex - pointillés rouges), qui sont corrélées. L'imagerie des vertex via les particules secondaires qui y sont émises, rayons gammas (bleu) et protons (rouge), permet donc de remonter à la distribution de la dose [2].

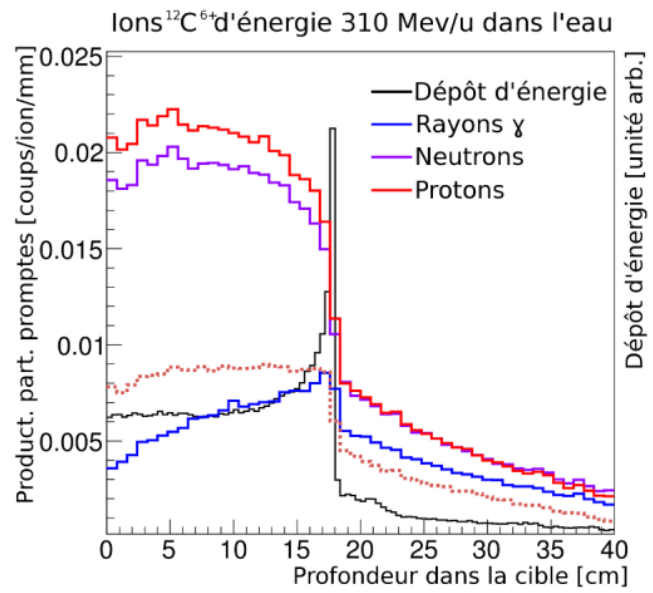

Figure 1 : distributions longitudinales des vertex, des particules promptes, et de la dose [2]

\subsection{3 État de l'art}

La tomographie par émission de positons a été utilisée en ligne au GSI-Darmstadt, et est actuellement utilisée hors ligne (après le traitement) en routine clinique dans certains centres [3]. Des recherches sont en cours pour une imagerie en ligne. Cette technique, qui repose sur l'imagerie des noyaux activés, est rendue complexe du fait de la lente décroissance radioactive de ces émetteurs. 


\section{Développements instrumentaux}

\subsection{Gamma caméra Compton}

\subsubsection{Principe}

Pour imager les rayons gamma, plusieurs types de caméras sont envisagées, dont la caméra collimatée et la caméra Compton [4] qui exploite l'effet du même nom, prédominant dans la gamme d'énergie des rayons gammas produits en hadronthérapie.

Lorsqu'un photon incident interagit sur un premier détecteur de la caméra (constitué de diffuseurs), il dépose une énergie en une position donnée, un photon de moindre énergie est alors réémis dans une autre direction avant d'être absorbé par un second détecteur (absorbeur). Connaissant les énergies déposées et les positions des deux interactions, il est possible de reconstruire le cône qui porte la trajectoire du photon incident.

Un principe de caméra Compton à temps de vol, constituée d'un empilement de diffuseurs silicium et d'un scintillateur absorbeur, est présenté Figure 2.

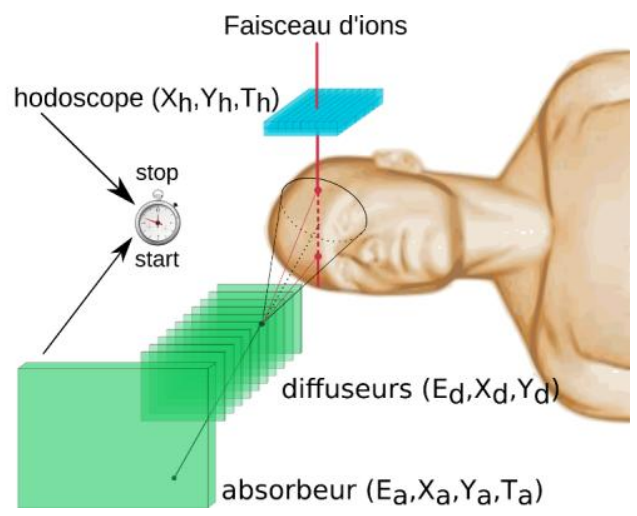

Figure 2 : principe de l'imagerie de vertex avec une gamma caméra Compton [4]

\subsubsection{Instruments développés}

\section{Dimensionnement des diffuseurs et de l'absorbeur}

Des simulations Monte-Carlo ont permis de dimensionner la caméra : position et nombre de diffuseurs (10) ainsi que leur géométrie $\left(10 \times 10 \times 0.2 \mathrm{~cm}^{3}\right)$ pour optimiser la statistique de reconstruction ainsi que la résolution de l'image obtenue [4].

\section{Principe des diffuseurs}

Les diffuseurs sont des détecteurs silicium dopés à bande double face. Lors de l'interaction d'un rayon gamma, des paires électrons-trous sont créées puis collectées par les bandes métallisées (64 par face), ce qui permet d'obtenir la position et l'énergie déposée.

\section{Electronique frontale}

La contrainte sur le grand nombre de voies du prototype (1280) ainsi que sur les performances en bruit (300 électrons RMS ramenés en entrée) ont motivé le développement d'un ASIC [5]. Les mesures réalisées valident son adéquation aux contraintes. 


\subsection{Trajectomètre de protons}

\subsubsection{Principe}

Pour imager les vertex à l'origine de protons secondaires [2], nous utilisons un trajectomètre constitué de quatre plans de deux détecteurs en silicium [6], développés pour la physique des hautes énergies. Ces détecteurs, de $2 \mathrm{~cm}^{2}$, sont pixélisés (1152x576 pixels), minces $(50 \mu \mathrm{m}$, limitant la diffusion des protons) et ont une exposition relativement rapide (112 $\mu \mathrm{s}$, temps mort négligeable). De plus, les données sont numérisées et compressées sur le détecteur.

Un logiciel a été développé pour analyser ces données : les pixels sont tout d'abord regroupés en amas, qui permettent l'extrapolation des traces, dont l'intersection avec la trajectoire de l'ion incident permet d'obtenir la position du vertex.

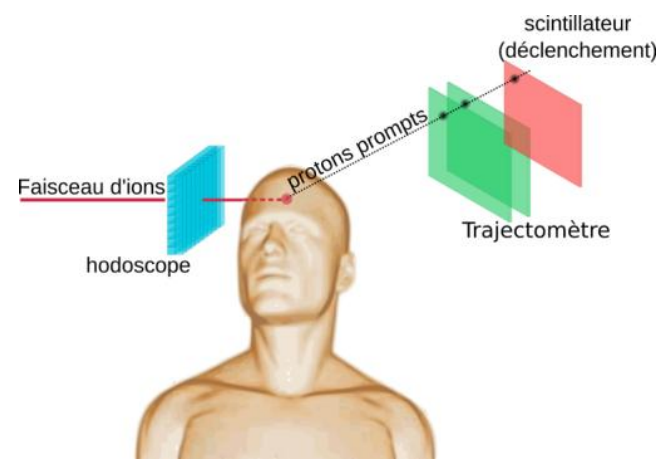

Figure 3 : principe de l'imagerie de vertex par protons (PIVI) en hadronthérapie carbone [2]

\subsubsection{Résultats}

Plusieurs expériences ont été réalisées : une cible en PPMA (10 × $\left.10 \times 25 \mathrm{~cm}^{3}\right)$ a été irradiée avec des ions carbone d'énergies typiques pour les traitements par hadronthérapie. Sur la figure 4 on observe les distributions longitudinales des vertex reconstruits pour quatre énergies. On remarque une corrélation avec les distributions théoriques de dose.

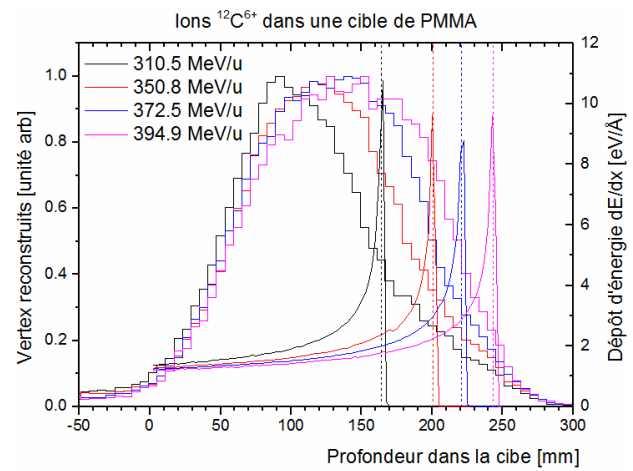

Figure 4 : Profils de dose et de vertex reconstruits obtenus avec la méthode PIVI 


\subsection{Hodoscope de faisceau}

\subsubsection{Principe}

L'hodoscope de faisceau est un instrument commun aux différentes méthodes d'imagerie présentées, et permet d'obtenir, pour chaque ion incident, sa position de passage (donc sa trajectoire) ainsi que son instant de passage (permettant l'association d'événements avec les autres instruments).

Notre prototype est constitué de deux couches croisées de fibres scintillantes carrées de $1 \mathrm{~mm}$, au nombre de 128 par couche. Lorsqu'un ion traverse l'hodoscope, il dépose une faible quantité d'énergie dans deux fibres croisées, créant ainsi de la lumière par scintillation. Un photomultiplicateur multivoies converti alors la lumière en un signal électrique.

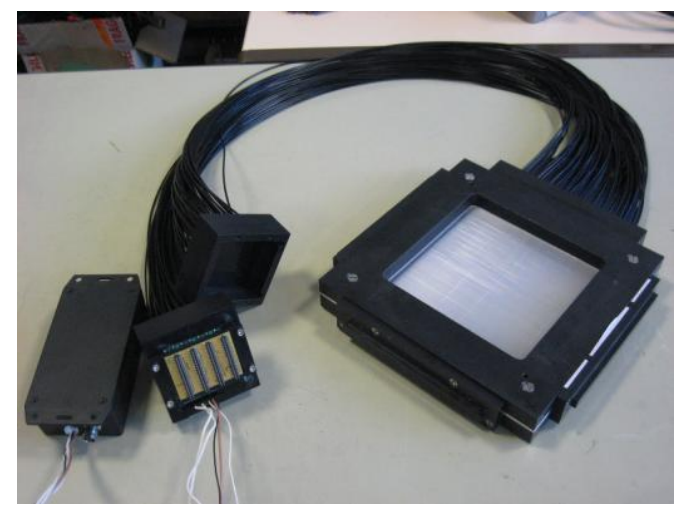

Figure 5 : prototype d'hodoscope $2 \times 128$ fibres, avec photomultiplicateur multivoies

\subsection{Contraintes}

Le système global, constitué de l'hodoscope, des photomultiplicateurs, et d'une électronique frontale développée spécifiquement (ASIC) est soumis à de fortes contraintes :

- Taux de comptage élevés souhaités : $10^{8}$ ions/s ;

- Résolution temporelle souhaitée : $1 \mathrm{~ns}$;

- Radiorésistance : plusieurs semaines en conditions cliniques.

\subsubsection{Electronique frontale}

Le nombre important des voies à analyser (2x128) ainsi que les contraintes citées ont motivé le développement d'ASIC spécifiques à cette application [7].

Tout d'abord, un ASIC « lecture » assure le traitement du signal électrique issu des photomultiplicateurs. Une structure « en courant » permet d'obtenir une amplification ainsi qu'une détection d'événements à forts taux de comptage : les tests en faisceaux ont validé le fonctionnement à $10^{8} \mathrm{~Hz}$.

De même, un circuit spécifique « mesure de temps » a été développé pour obtenir une résolution temporelle de $1 \mathrm{~ns}$. Une boucle à verrouillage de délais permet, via 32 étages à $160 \mathrm{MHz}$, d'obtenir une résolution temporelle de $195 \mathrm{ps}$. Une gigue de $15 \mathrm{ps}$ a été mesurée.

Une nouvelle version d'ASIC intégrant les deux circuits est en cours de réalisation. 
V. Reithinger et al.

\section{Conclusion et perspectives}

L'hadronthérapie est une technique de thérapie novatrice qui motive le développement d'instruments pour le suivi en ligne des traitements. Ces instruments innovants sont soumis à de fortes contraintes telles que des taux de comptage élevés, et illustrent les retombées technologiques de la physique des hautes énergies au domaine médical.

Les instruments présentés, prototypes fonctionnels ou en cours de réalisation, sont en cours de tests, avec une évaluation en conditions cliniques (fantômes réalistes) prévue d'ici à deux ans.

\section{Remerciements}

\section{Collaborateurs}

Centre Antoine-Lacassagne (Nice, France) : J. HERAULT ; Institut de Physique Nucléaire d'Orsay (Orsay, France) : M. CHABOT ; GSI Helmholtzzentrum für Schwerionenforschung (Darmstadt, Allemagne) : C. LA TESSA, R. PLESKAC, M. VANSTALLE ; Institut Pluridisciplinaire Hubert Curien (Strasbourg, France) : C. FINCK, R. RESCIGNO, M. ROUSSEAU ; Ion Beam Application SA (Louvainla-Neuve, Belgique) : D. PRIEELS, F. ROELLINGHOFF, J. SMEETS, F. STICHELBAUT ; Laboratoire de Physique Corpusculaire (Clermont-Ferrand, France) : P. FORCE, B. JOLY ; University Clinic Heidelberg (Heidelberg, Allemagne) : S. BRONS, K. PARODI, I. RINALDI

\section{Supports financiers}

QAPIVI (Inserm) ; PRRH (CPER Région Rhône-Alpes) ; ENVISION (Union Européenne), ENTERVISION (programme Marie-Curie, Union Europ.) ; GdR MI2B (CNRS-IN2P3) ; GamHadron (ANR) ; Labex PRIMES, Labex LIO

\section{Bibliographie}

[1] Schardt D, Elsässer T, Schulz-Ertner D. Heavy-ion tumor therapy: Physical and radiobiological benefits. Rev. Mod. Phys. 2010 Feb 19;82(1):383-425.

[2] Henriquet $P$, Testa $E$, et al. Interaction vertex imaging (IVI) for carbon ion therapy monitoring: a feasibility study. Physics in Medicine and Biology. 2012 Jul 21;57(14):4655-4669.

[3] Parodi K, Bortfeld T, et al. PET imaging for treatment verification of ion therapy. Nuclear Instruments and Methods in Physics Research Section A. 2008 Jun 11;591(1):282-286.

[4] Roellinghoff $F$, Richard M-H, et al. Design of a Compton camera for 3D prompt gamma imaging during ion beam therapy. Nuclear Instruments and Methods in Physics Research Section A. 2011 Aug;648:S20-S23.

[5] Dahoumane M, Dauvergne D, et al. A Low Noise and High Dynamic Charge Sensitive AmplifierShaper associated with Silicon Strip Detector for Compton Camera in hadrontherapy. 2012 IEEE Nuclear Science Symposium and Medical Imaging Conference (NSS/MIC). Available from: http://hal.in2p3.fr/in2p3-00753748

[6] Hu-Guo C, Baudot J, et al. First reticule size MAPS with digital output and integrated zero suppression for the EUDET-JRA1 beam telescope. Nuclear Instruments and Methods in Physics Research Section A. 2010 Nov 1;623(1):480-482.

[7] Deng S, Dauvergne D, et al. Very fast front end ASIC associated with multi-anode PMTs for a scintillating-fibre beam hodoscope. Journal of Instrumentation. 2013 Jan 30;8(01):C01047C01047. 